\title{
„Das Gesundheitswesen sollte Willkommenskultur stärker pflegen und systematisieren"
}

„Willkommenskultur in der Radiologie geht das überhaupt“? Unter diesem Titel fand auf dem 102. Deutschen Röntgenkongress eine von der internen Kommission Diversity@DRG der Deutschen Röntgengesellschaft organisierte Veranstaltung statt. Dabei mitdiskutiert hat auch Prof. Dr. Konstantin Nikolaou, DRG-Vorstandsmitglied und Ärztlicher Direktor der Abteilung für Diagnostische und Interventionelle Radiologie im Universitätsklinikum Tübingen. Wir haben uns mit Professor Nikolaou über Willkommenskultur in der Radiologie und im Gesundheitssystem sowie über Strukturen und Prozesse für eine gute Willkommenskultur unterhalten.

\section{Herr Professor Nikolaou, was gibt es beim Thema Willkommenskultur aus radiologi- scher Perspektive zu berichten?}

Zunächst einmal freue ich mich sehr, dass die Veranstaltung „Willkommenskultur in der Radiologie - geht das überhaupt?“ stattgefunden hat, denn es handelt sich dabei um ein Thema, das nicht immer sehr präsent ist, über das es sich aber lohnt, aktiv nachzudenken. Generell geht es beim Thema „Willkommenskultur" nicht unbedingt nur um eine spezielle radiologische Perspektive in Kliniken oder Praxen, sondern um eine Systematik des „Willkommenen Heißens“ im Gesundheitssystem insgesamt. Ich denke, dass bei diesem Thema andere Branchen zum Teil schon weiter

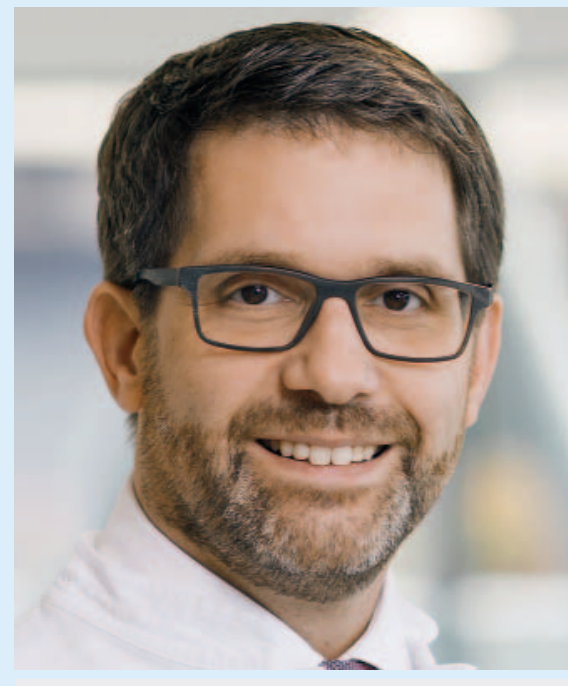

Prof. Dr. Konstantin Nikolaou 
sind. Doch auch das Gesundheitswesen sollte Willkommenskultur stärker pflegen und systematisieren.

Willkommenskultur umfasst viele Aspekte: Zum Beispiel Prozesse der Einarbeitung und des Onboardings von neuen Mitarbeiterinnen und Mitarbeitern oder ihre Integration in Kliniken und Praxen. Was macht für Sie eine gute Willkommenskultur aus?

Willkommenskultur zielt darauf ab, dass neue Mitarbeitende gut ankommen, schnell in die Arbeit finden und von Beginn an effektiv arbeiten können. Dabei gehört zu einer guten Willkommenskultur eine persönliche Ebene, sozusagen ein emotionales Ankommen und ein Sich-betreut-fühlen. Zu einer guten Willkommenskultur gehören aber natürlich auch formale und praktische Aspekte. Dazu zählen Strukturen, Abläufe und Routinen, über die klar wird, was bei der Einarbeitung neuer Mitarbeitender in welcher Reihenfolge geschieht. Es gibt also die Sachebene und die emotionale Ebene. Ich finde die emotionale Ebene fast noch wichtiger, weil ich glaube, dass der erste Eindruck und wie eine Person in ein Team kommt, wie sie dort wahrgenommen wird und auch wie sie selbst die neue Umgebung und Situation empfindet, viel dazu beiträgt, wie neue Mitarbeiterinnen und Mitarbeiter in ihre Arbeit finden. Deshalb sollten sich Arbeitgeberinnen und Arbeitgeber sowie Führungskräfte insgesamt mit großer Sorgfalt um neue Mitarbeitende kümmern - gerade auch, weil es sich bei ihnen oft um Studienabgängerinnen und Studienabgänger handelt, die am Beginn ihres Arbeitslebens stehen und noch nicht viele beruflichen Erfahrungen mitbringen.

Sie sind ärztlicher Direktor der Abteilung für Diagnostische und Interventionelle Radiologie im Universitätsklinikum Tübingen. Wie leben und gestalten Sie in Ihrer Abteilung oder generell im Universitätsklinikum „Willkommenskultur“?

Im Universitätsklinikum Tübingen gibt es strukturierte Standardabläufe beim Onboarding, etwa mit Einführungstagen für neue Mitarbeiterinnen und Mitarbeiter, auf denen Grundsätzliches erklärt wird, wo man zum Beispiel erste Kontakte knüpfen oder die IT-
Strukturen kennenlernen kann. Bei uns in der Abteilung haben wir eine Einarbeitungsrotation entwickelt und etabliert, in der neue Mitarbeiterinnen und Mitarbeiter zunächst alle wichtigen Bereiche durchlaufen, um diese und das Team kennenzulernen. Erst dann lernen die „Neuen“ die Details ihres spezifischen Arbeitsplatzes kennen. Neue Mitarbeitende bekommen von uns eine Einarbeitungsmappe mit Informationen zu allen wichtigen Standardfragen, die sich am Anfang stellen: Informationen zur Abteilung und zum Organigramm, Regeln bezüglich Urlauben und Dienstreisen, wichtige Ansprechpartnerinnen und Ansprechpartner und vieles mehr.

Um die emotionale Ebene abzudecken, enthält der Prozess der Einarbeitung bei uns verschiedene Elemente: Er beginnt schon einige Wochen vor dem Arbeitsstart mit einer ersten E-Mail, in der wir künftige Mitarbeitende fragen, ob sie etwas brauchen, wann es am ersten Arbeitstag los geht und wie der Ablauf dieses Tages sein wird. Wenn der oder die „Neue“ dann bei uns anfängt, stellen wir ihm oder ihr eine Mentorin oder einen Mentor zur Seite. Als Mentorin oder Mentor fungieren bei uns erfahrene Assistentinnen oder Assistenten, die sich um die neuen Mitarbeitenden kümmern und hauptsächliche Ansprechpartnerinnen und Ansprechpartner in den ersten Monaten sind.

Ich selber verabrede mit neuen Kolleginnen und Kollegen relativ schnell ein erstes Kaffeetrinken, um sie zu begrüßen, ein erstes Feedback einzuholen und zum Beispiel zu erfragen, wie es läuft - am Arbeitsplatz in der Klinik, aber auch in der Stadt Tübingen, wenn die neuen Mitarbeitenden dorthin gezogen sind. Ich finde das Feedback von Mitarbeitenden nicht nur direkt am Anfang, sondern über die gesamte Einarbeitungszeit wichtig, denn man lernt so die Perspektive der anderen Person kennen und man erfährt, was man als Arbeitgeberin oder Arbeitgeber besser machen kann. Insgesamt dauert der Prozess der Einarbeitung drei Monate und der des Mentoring etwa sechs Monate, also eine relativ lange Zeit.

Wenn Sie auf Ihren beruflichen Lebensweg zurückblicken - welche Formen von Willkommenskultur haben Sie persönlich kennengelernt?
Als ich vor ungefähr 20 Jahren als Assistenzarzt angefangen habe, gab es noch keine wirklich systematische Willkommenskultur, sicherlich aber einige Elemente davon. Aber ich hatte das Glück, in einer sehr netten Abteilung anzufangen und diese durch meine Promotion auch schon zu kennen, sodass ich jederzeit mit den Kolleginnen und Kollegen sprechen und mich problemlos durchfragen konnte, wenn ich etwas brauchte. Ein bisschen hat es sich ab und zu schon wie das sprichwörtliche „kalte Wasser" angefühlt, wenn ich etwa meinen Arbeitsplatz früh allein gestalten musste. Aber insgesamt hat alles sehr gut geklappt. Ich war als junger Assistent auch einmal ein Jahr in den USA im Auslands-Fellowship. Dort gab es schon eine in meinen Augen etwas geregeltere Willkommenskultur, in der man eher strukturiert eingewiesen wurde.

Würden Sie sagen, dass die USA das Thema schon früher als Deutschland für sich entdeckt haben?

Ich habe nur eine punktuelle Erfahrung gemacht und kann das daher nicht im Allgemeinen für die USA beurteilen. Bei dem speziellen Institut, an dem ich tätig war, gab es einen klaren Fahrplan für die Einarbeitung und das Onboarding neuer Mitarbeiterinnen und Mitarbeiter. Was ich in Deutschland und in Europa aber seit einiger Zeit beobachte, ist, dass Fragen der Willkommenskultur stärker diskutiert werden und dass es ein immer größeres Bewusstsein dafür gibt, dass die besten und nettesten Mitarbeiterinnen und Mitarbeiter nicht unbedingt von alleine durch die Tür kommen oder für immer in einer Abteilung oder einer Klinik bleiben. Auch im Gesundheitssystem gibt es einen Fachkräftemangel und insofern müssen Arbeitgeberinnen und Arbeitgeber aktiv werden, um Mitarbeitende werben, sich um sie bemühen und ihnen wertschätzend begegnen.

Denken Sie, dass man Willkommenskultur lernen kann?

Ich denke schon, dass man Willkommenskultur, etwa durch die Anschauung von Best Practice-Beispielen, erlernen kann. Von solchen Beispielen kann man für sich und die Willkommenskultur in der eigenen Abteilung oder der Klinik nützliche Elemente und Bausteine übernehmen. Außer- 
dem gibt es Literatur zum Thema ebenso wie Fortbildungen. Wie die emotionale Ebene gestaltet wird, ist natürlich individuell sehr verschieden. Da müssen Arbeitgeberinnen und Arbeitgeber ihren eigenen Stil finden, der dann auch die Kultur in der jeweiligen Abteilung oder Klinik insgesamt prägt.
Wie kann man die Wahrnehmung und das Bewusstsein für eine Willkommenskultur stärken?

Ich finde es sehr gut und nützlich, dass das Thema zum Beispiel auf dem Deutschen Röntgenkongress aufgegriffen wurde. Entsprechende Veranstaltungen sind ein wichti- ger Schritt hin zu mehr Sensibilität für das Thema. Darüber hinaus wird über solche Veranstaltungen ja auch ein Dialog organisiert und initiiert, in dem man sich über Erfahrungen und Notwendigkeiten austauschen und Beispiele und Erkenntnisse vermitteln kann. Insofern wünsche ich mir, dass es in Zukunft mehr solcher Veranstaltungen gibt. 